\title{
A Case Report on the Risk of Enterobacteriaceae Infection in the Oral and Maxillofacial Region
}

\author{
Lee-Rang Lim', Young-Cheol Lee', Hye-Jung Lee', Gyeo-Woon Jung', \\ $\mathrm{Na}-\mathrm{Ra}$ Yun ${ }^{2}, \mathrm{Yo}-\mathrm{Seob} \mathrm{Seo}^{3}$, Ji-Su Oh${ }^{1}$, Jae-Seek You' \\ 1Department of Oral and Maxillofacial Surgery, School of Dentistry, Chosun University, Gwangju, Korea \\ ${ }^{2}$ Division of Infectious Disease, Department of Internal Medicine, College of Medicine, Chosun University, Gwangju, Korea \\ ${ }^{3}$ Department of Oral and Maxillofacial Radiology, School of Dentistry, Chosun University, Gwangju, Korea
}

Received August 13, 2019

Revised September 6, 2019

Accepted September 8, 2019

\section{Correspondence to:}

Jae-Seek You

Department of Oral and Maxillofacial

Surgery, School of Dentistry, Chosun

University, 309 Pilmun-daero, Dong-gu,

Gwangju 61452, Korea

Tel: +82-62-220-3816

Fax: +82-62-222-3810

E-mail:applit375@chosun.ac.kr

https://orcid.org/0000-0001-7638-9583
Osteomyelitis is an inflammatory condition of the bone caused by pathogenic bacteria. The causative pathogen is usually oral residing bacteria, but this is a report of patients with osteomyelitis infected with Enterobacteriaceae, which is not common. Enterobacteriaceae has been known to cause in-hospital infections for over last 30 years and is known to have multiple antibiotic resistances. Both cases in this study developed osteomyelitis after removal of the dentigerous cyst. Enterobacter aerogenes was cultured in one patient and Serratia marcescens in the other. After changing antibiotics through antibiotic susceptibility testing, clinical symptoms subsided and radiographic images confirmed that the callus formed and recovered at the same time.

Key Words: Enterobacter aerogenes; Osteomyelitis; Serratia marcescens

\section{INTRODUCTION}

Osteomyelitis of the jaw is a sign of acute or chronic inflammation of the cancellous bone or cortical bone. In children, osteomyelitis occurs primarily hematogenous in origin and acute in nature. On the other hand, in adults, osteomyelitis usually occurs as a subacute or chronic infection and most of them are damaged in bone and peripheral soft tissues. Bacteria isolated from osteomyelitis caused by infection are often associated with the patient's age or trauma and recent surgery [1]. The main etiology is bacteria's presence in oral cavity [2]. Stapylococcus aureus is the most common cause of acute and chronic osteomyelitis but osteomyelitis is also associated with many other pathogenic bacteria [3,4]. In addition, systemic diseases, alcohol, immunosuppression, diabetes and hypovascular bone disease (osteopetrosis, Paget's disease, florid cemento-osseous dysplasia, radiotherapy, etc.) are factors that increase the chance of osteomyelitis [4,5]. In general, antibiotic therapy is the basis of osteomyelitis treatment, but if there is no clinical improvement, surgical approach through curettage or resection of the infected area is required [6].

The patients reported are a case of osteomyelitis caused by Enterobacter aerogenes and Serratia marcescens. The common feature between $E$. aerogenes and $S$. marcescens is that they belong to Enterobacteriaceae. Enterobacteriaceae is a very large family that includes 22 genus populations including Escherichia, Shigella, and Salmonella which are usually present in intestines of human and animals and are important causes of infection in the intestinal tract. These are facultative anaerobe, characterized by Gram negative bacillia and are cultured separately using MacConkey agar. They are originally found in water and soil and usually in the gut microbiota in humans and other animals. Particularly, they are separated from cerebrospinal fluid, synovial fluid and abscess and hospital-acquired infection by

Copyright (c) 2019 Korean Academy of Orofacial Pain and Oral Medicine. All rights reserved.

(c) This is an open-access article distributed under the terms of the Creative Commons Attribution Non-Commercial License (http://creativecommons.org/licenses/by-nc/4.0/), which permits unrestricted non-commercial use, distribution, and reproduction in any medium, provided the original work is properly cited. 
antibiotic-resistant bacteria, such as $\beta$-lactamase, carbapenamases and fluoroquinolones can cause serious problems [7].

Based on previous literature review, osteomyelitis caused by $E$. aerogenes and $S$. marcescens is extremely rare in adults without drug infusion or other direct exposures [810]. Osteomyelitis caused by $S$. marcesces after leg or spinal surgery, or gunshot wounds is often reported, but a case of osteomyelitis caused by $S$. marcescens after oral and maxillofacial surgery has been found in only one paper, but none of osteomyelitis by $E$. aerogenes were found [11-15].

This report describes a case of osteomyelitis caused by infection of $E$. aerogenes and $S$. marcescens in patients with a removed dentigerous cyst including a mandibular third molar.

\section{CASE REPORT}

\section{Case 1}

A 53-year-old man presented with a chief complain of a cystic lesion including the left lower third molar to department of oral and maxillofacial surgery, Chosun University Dental Hospital on September 7, 2016. There was no
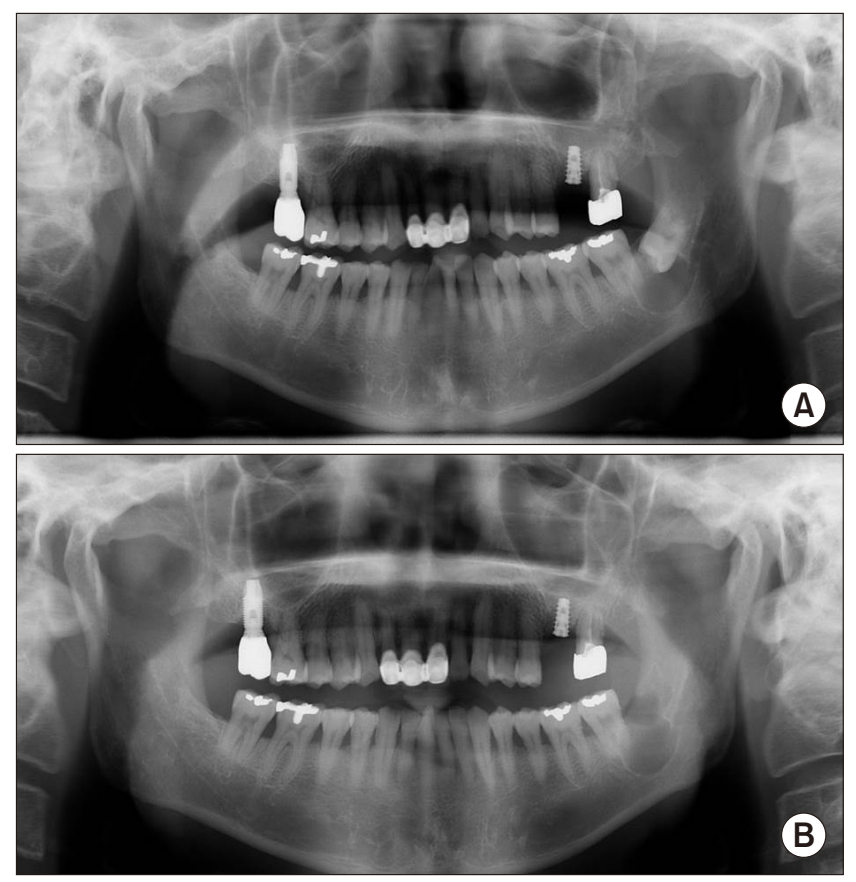

Fig. 1. Case A. Panoramic radiograph before (A) surgery and (B) 4 days after surgery. medical history and subjective complaints, such as discomfort or pain. Cone beam computed tomography (CBCT) was taken to confirm the size and appearance of the lesion, and the radiolucency with definite hyperostotic border was observed around the crown of horizontally impacted left lower third molar. It was also confirmed that the left mandibular canal was displaced downward. Based on clinical and radiological examination, the patient was diagnosed as a cystic lesion including the lower left third molar and planned for extraction of the tooth and removal of the cyst under general anesthesia.

On October 11, 2016, under the general anesthesia, an oral approach was performed, with vertical incision on the mesial side of the left lower second molar and crestal incision on the distal side. After the extraction of the left lower third molar, the cyst was removed. At the site where the cystic lesion was removed, collagen plug (Ateloplug; Bioland, Cheongwon, Korea) was inserted and primary suture was performed. The tissue from the pathological site was referred for histopathological examination. An antibiotic agent (augmentin; Ilsung Pharma Co., Seoul, Korea, 1.2 g, 3 times a day) was injected into the vein during admission. Four days after the operation, he was discharged from the hospital and was prescribed oral antibiotics (augmentin $625 \mathrm{mg}, 2$ times a day). Removal of suture, stitch out was performed 7 days later. It was diagnosed as a dentigerous cyst through histopathological examination (Fig. 1).

Four weeks after the surgery, the patient complained of swelling and pain in the operation site, and the surgical site was opened and bone exposure was observed. However, pus was not released and no radiolucency around the surgical site was observed in the panoramic image (Fig. 2).

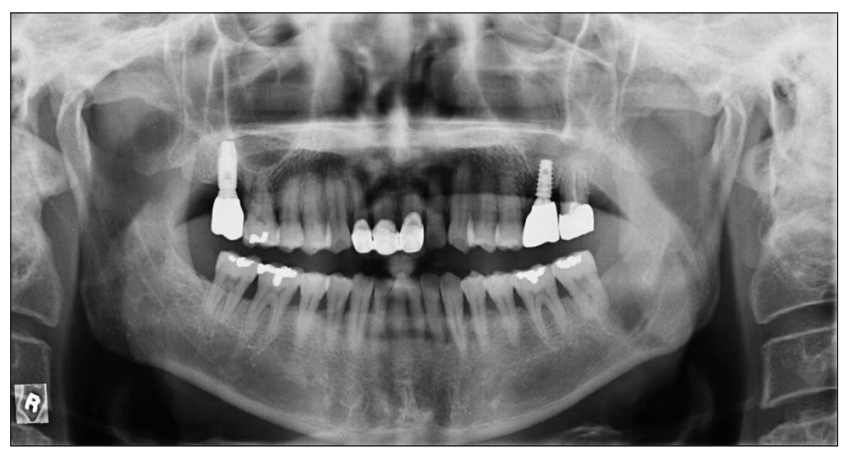

Fig. 2. Case A. Panoramic radiograph 4 weeks after surgery. 

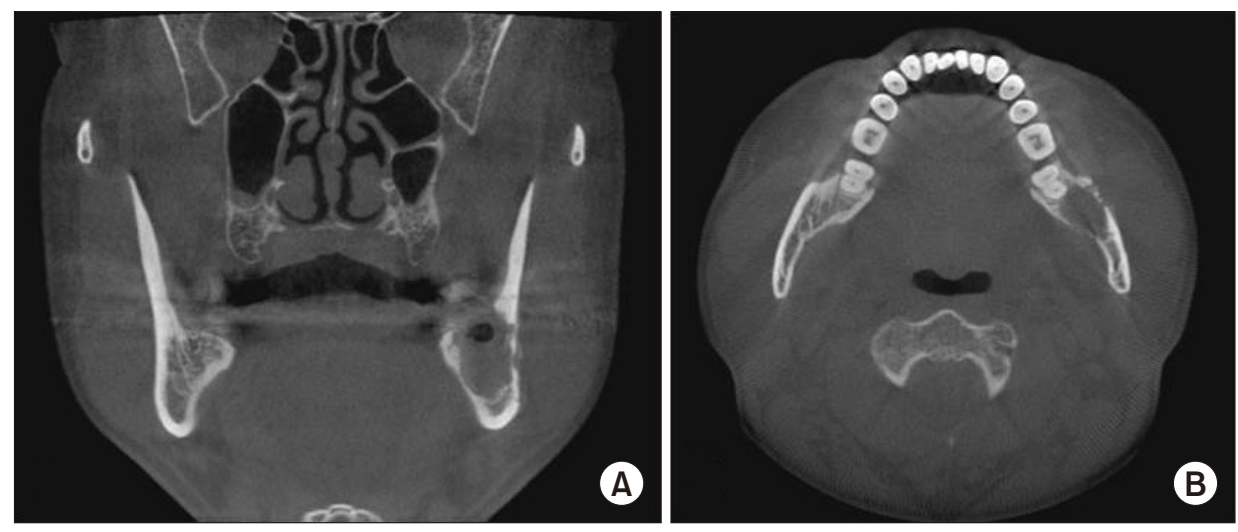

Fig. 3. Case A. (A) Coronal view and (B) axial view of cone beam computed tomography images 6 weeks after operaion. Destroyed cortical bones around the surgical site.

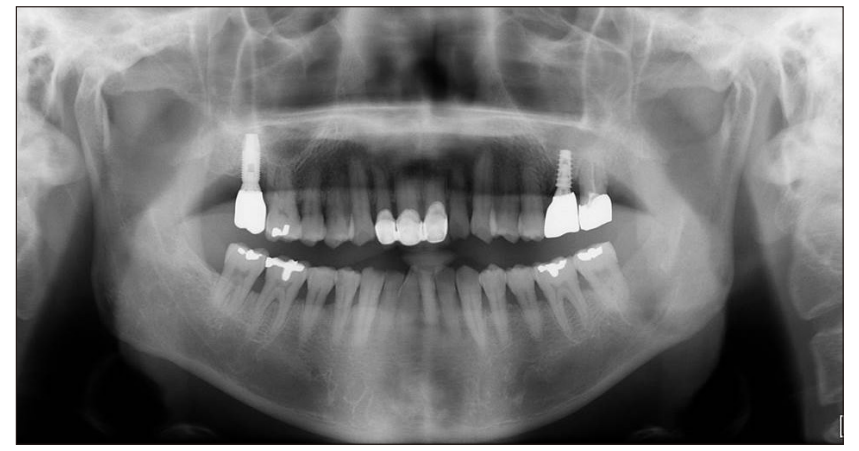

Fig. 4. Case A. Panoramic radiograph 5 months after operation. No additional bone destruction were observed, but bone formation was observed.

Additional antibiotics were prescribed and planned for follow-up. CBCT was taken because the symptoms did not improve for two weeks, and the images confirmed bone destruction around the extraction socket (Fig. 3). The necrotic tissue was removed under local anesthesia and the collected tissue was referred to laboratory for bacterial culture. The patient was hospitalized and augmentin was injected intravenously (1.2 g, 3 times a day). Additional panoramic images and CBCT were taken during admission, but bone destruction was further developed around the left mandibular canal and left mandibular angle. E. aerogenes were detected from the tissue bacterial culture test and cefepime (Maxipime; Boryung, Seoul, Korea, 2 g, twice daily) was injected intravenously for two weeks based on antibiotic sensitivity test results.

Panoramic radiograph and CBCT images taken 7 weeks after surgery confirmed that bone destruction had not progressed further and the patient's discomfort was reduced. Five months after the surgery, radiographic images confirmed the formation of the callus and the progression of
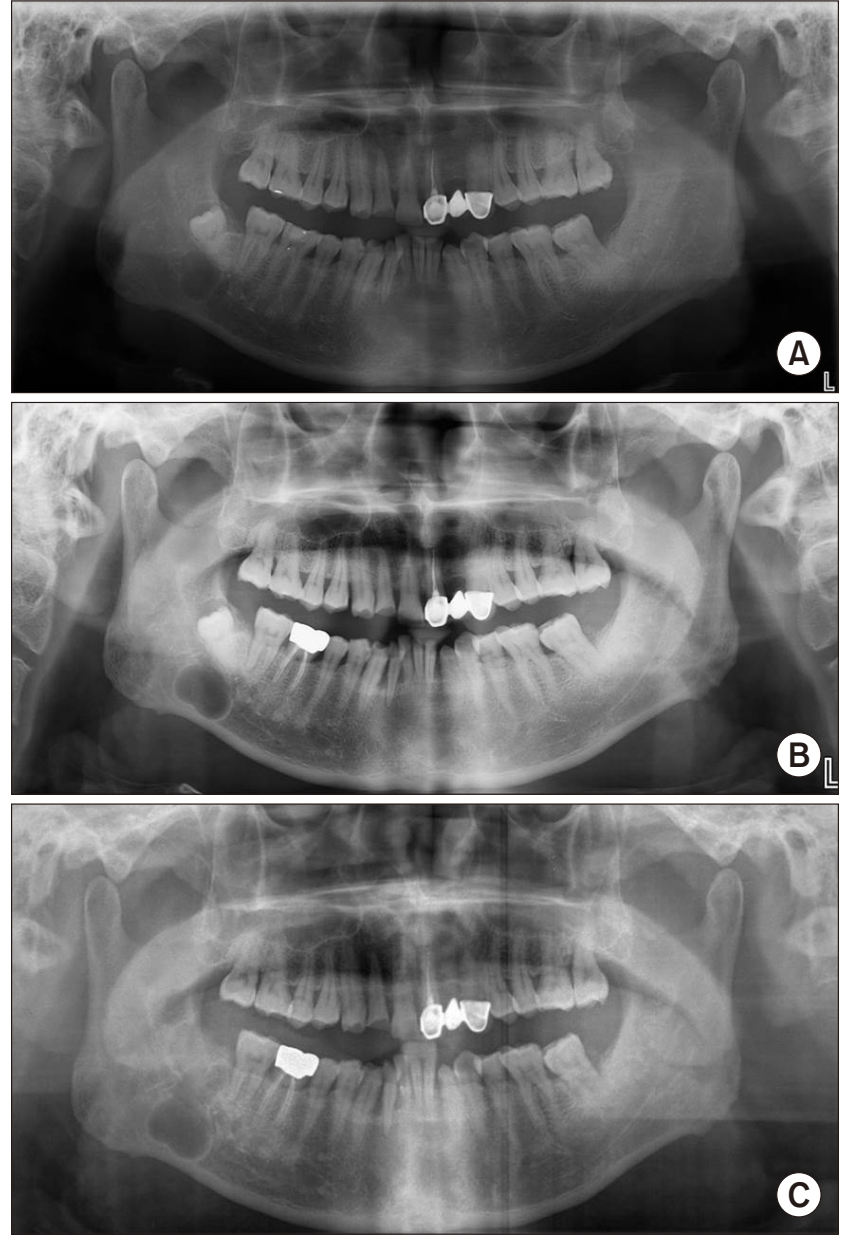

Fig. 5. Case B. Panoramic radiograph before in (A) 2010 and (B) 2018 and $(C)$ immediately after surgery.

bone remodeling (Fig. 4).

\section{Case 2}

A 57-year-old man presented with a chief complain of a cystic lesion including the right lower third molar to Oral and Maxillofacial Surgery, Chosun University Dental 
Hospital on July 6, 2018. He visited another dental clinic to treat tooth attrition before, but was referred to the hospital for further evaluation and treatment after the panoramic radiograph was taken. The patient was taking medication for hypertension and had no other medical history. The patient did not complain of any particular discomfort or paresthesia. There were no findings of sinus tract or infection in intraoral and extraoral area.

A cystic lesion was also observed in the panoramic image taken on May 20, 2010, but only endodontic and prosthetic treatments were performed for the right lower first molar. In the panoramic image taken on July 6, 2018, the cystic lesion below the right lower third molar was larger than in the previous panoramic image (Fig. 5). CBCT was taken to confirm the size and extent of the lesion. CBCT images showed radiopaque area inside the radiolucency of the definite hyperostotic border below the impacted right lower third molar, and confirmed that the lingual cortical bone got thin and there was a slight expansion. In addition, inferior border of the right mandibular canal was displaced to the buccal and upper side of the lesion, and the continuity of the inferior border was lost. There were pericoronal radiolucency surrounding the mandibular third molar and a fistula from the crown of mesial side to the the distocrestal area of the mandibular second molar. Based on clinical and radiological examination, the patient was diagnosed as a cystic lesion including the lower right third molar and planned for extraction of the tooth and removal of the cyst under general anesthesia.

On August 21, 2018, under general anesthesia, after the extraction of the right lower third molar and removal of the cyst, at the site where the lesion was removed, collagen plug (Ateloplug) and steroid (Dexamethasone; Yuhan, Seoul, Korea) was applied and primary suture was performed. The tissue from the pathological site was referred for histopathological examination. The patient was given intravenous antibiotics (augmentin $1.2 \mathrm{~g}, 3$ times a day) during admission, and oral antibiotics (augmentin $625 \mathrm{mg}$, twice a day) were prescribed from the day after discharge. Removal of suture, stitch out was performed 7 days later. It
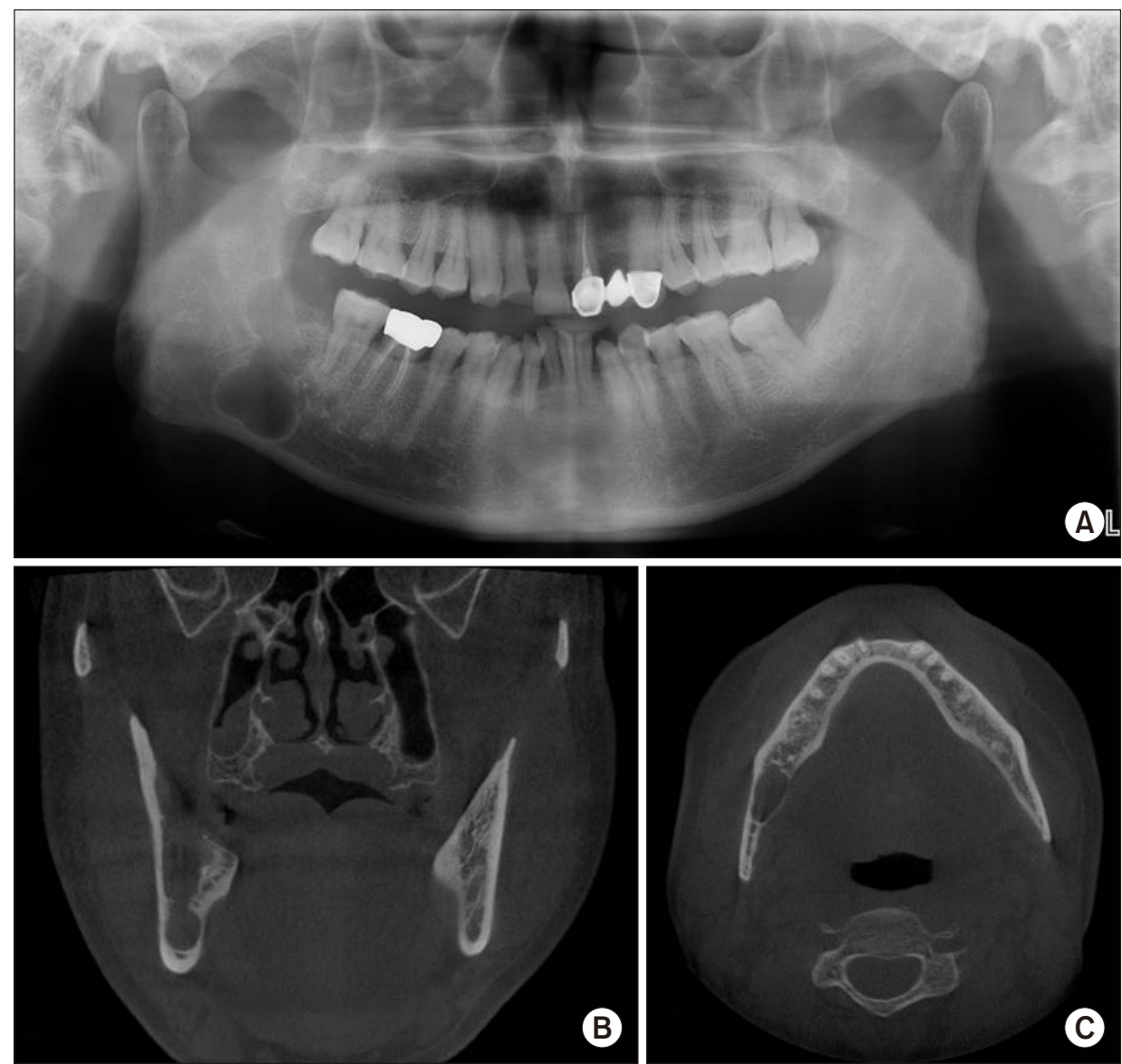

C
Fig. 6. Case B. Panoramic (A) radiograph, (B) coronal view and (C) axial view of cone beam computed tomography images three weeks after surgery. No specific change is observed. 
was diagnosed as a dentigerous cyst through histopathological examination.

Three weeks after the operation, sinus tract was observed in the buccal gingiva of the right lower first molar, and it was confirmed that it started at the operation site by guttapercha cone tracing. Panoramic radiograph and CBCT were taken and no unusual changes were observed (Fig. 6). An incisional biopsy was performed under local anesthesia and additional antibiotics (augmentin $625 \mathrm{mg}$, twice daily) were prescribed. Histopathological examination revealed inflammatory granulation tissue. No further clinical improvement was seen for 4 weeks and additional CBCT was taken due to pain complaints. No further clinical improvement was seen for 4 weeks and additional CBCT was taken due to sustained pain. Osteolysis changes were observed on CBCT images due to the loss of continuity of buccal cortical bone at the site of operation (Fig. 7). The necrotic tissue was removed under local anesthesia, and the removed tissue was requested for bacterial culture test. $S$. marcescens was detected in the bacterial culture test and moxifloxacin (Avelox; Bayer Korea Ltd., Seoul, Korea, 400 mg, once daily) was prescribed orally for 10 weeks based on antibiotic sensitivity test results. It was found that callus was formed through CBCT taken three weeks after the medication of moxifloxacin, and that the continuity of cortical bone was recovered in the images taken three months later (Fig. 8). In addition, the patient's symptoms disappeared. So, we decided to stop administrations of antibiotics and follow-up.

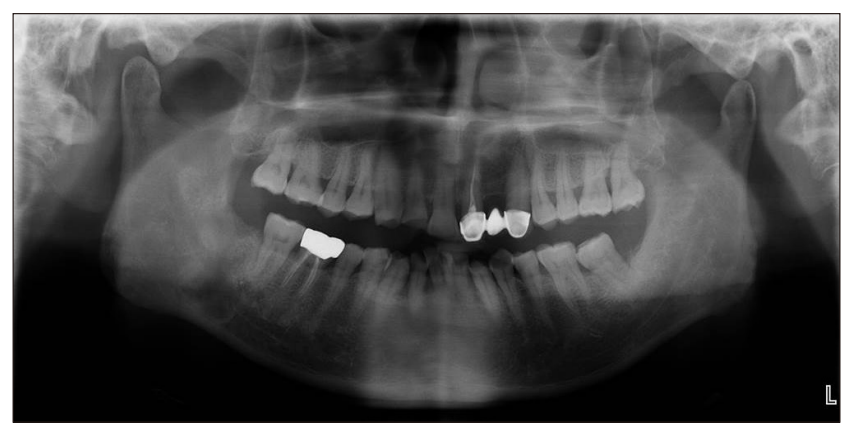

Fig. 8. Case B. Panoramic radiograph 4 months after surgery. Radiopacity was increased at the operation site and bone formation was observed.
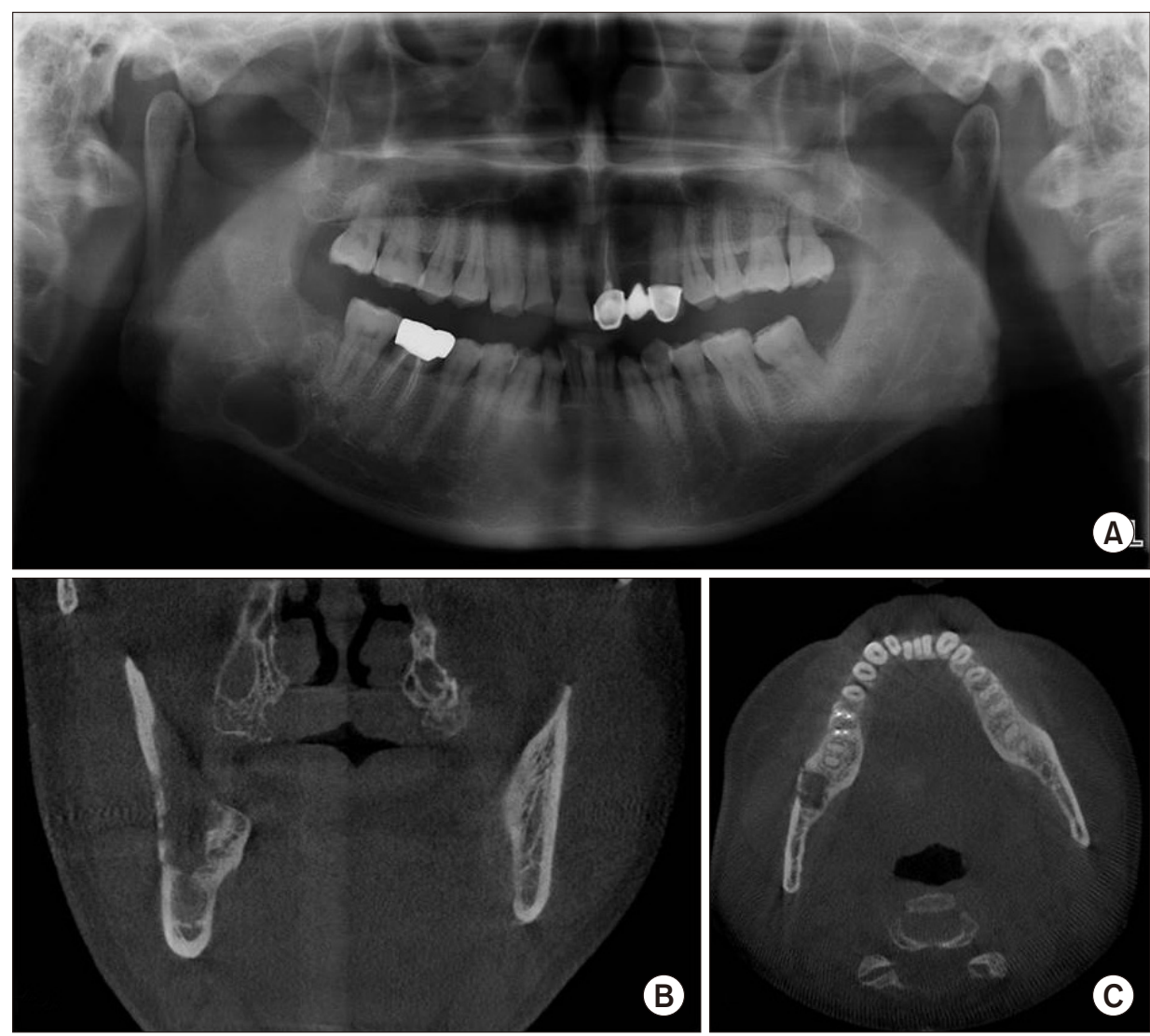

Fig. 7. Case B. Panoramic (A) radiograph, (B) coronal view and (C) axial view of cone beam computed tomography images 7 weeks after surgery. Cortical bone destruction around the operation site is observed. 


\section{DISCUSSION}

Osteomyelitis is a term that leads to bone disease caused by inflammatory reactions, and bone necrosis, suppuration, bone resorption, and bone sclerosis are observed [16,17]. The most common causes of osteomyelitis are bacterial and are also caused by dental infections, post-extraction complications, inadequate removal of necrosis bone, early discontinuation of antibiotics, incorrect selection of antibiotics, incorrect diagnosis, trauma, inadequate treatment after trauma, radiotherapy of jaw bones, etc. [18]. The two cases reported can be seen as osteomyelitis after removal of dentigerous cysts and tooth extraction.

Clinical features of osteomyelitis include pain, fever, swelling, sinus tract or fistula connected to intra or extra oral area, delayed healing, pathological fractures, and mouth opening limitations, etc. In case 1 , the dehiscence of the operation site due to delayed healing was observed, and in case 2, intraoral sinus tract was observed.

Radiologic examination can be used to observe radiolucency, bone destruction and sequestrum formation and through these, can diagnose osteomyelitis [16]. Taking a CBCT can provide a well-defined image of tissue calcification. In addition, sclerosis and loss of trabecular bone, changes in width and height of a bone, and perforation of cortical bone may be observed [19]. In these cases, radiolucency and perforation of cortical bone were clearly seen in the bone defect of the operation site, which were diagnosed as osteomyelitis.

The two patients were diagnosed with osteomyelitis based on pain, suppurative lesions, and bone destruction on CBCT. Conservative treatment was performed by curettage of the lesion. Tissue samples obtained by curettage were referred for histopathological examination and bacterial culture. Histopathological examination revealed a chronic inflammatory condition with fibrosis and an inflammatory granulation tissue. E. aerogenes were detected in case 1 patient and $S$. marcescens in case 2 patient.

E. aerogenes is gram-negative bacteria that is included in the genus Enterobacter and is also named Klebsiella aerogenes. It is usually found in the gastrointestinal tract of a human, and is an in-hospital infection that does not cause disease in healthy people but often causes opportunistic infections [20]. Infections with E. aerogenes often occur during intravenous or surgical procedures and are considered to be important multidrug-resistant pathogens, especially in the intensive care unit [21]. It is mainly separated from the respiratory tract, urinary tract, blood and intestinal tract [22]. They are susceptible to cefotaxime, cefepime, ceftazidime, ertapenem, gentamicin, ciprofloxacin, aztreonam in antibiotic sensitivity test.

Genus Serratia is a gram-negative bacterium that has been first identified as a pathogen that can cause serious infections in human since mid-1960 and accounts for $1.03 \%$ of hospital acquired infections [23,24]. In particular, $S$. marcescens is found mainly in showers, toilets and wet tiles, and is the only pathogen among species Serratia. Infection in humans causes sporadic urinary tract infections and pneumonia, and is often caused in patients who have been in the intensive care unit for a long time [25,26]. Cases of osteomyelitis caused by $S$. marcescens infection in adults are associated with extrinsic exposure, such as intravenous drug injection or surgical site, trauma, intra-articular injection. The bacterium has antibiotic susceptibility to cefotaxime, cefepime, ceftazidime, ertapenem, gentamicin, ciprofloxacin, aztreonam.

In both patients, according to the antibiotic sensitivity test, the symptoms disappeared after receiving cefepime for case 1 patient and moxifloxacin for case 2 patient. And bone remodeling was observed on CBCT.

The case of two patients reported that they might be infected with $E$. aerogenes and $S$. marcescens during hospitalization after oral and maxillofacial surgery. Attention should be paid to Enterobacteriaceae infections, because bone destruction, symptoms of osteomyelitis, may persist despite the continued medications of antibiotics.

\section{CONFLICT OF INTEREST}

No potential conflict of interest relevant to this article was reported.

\section{ORCID}

Lee-Rang Lim

https://orcid.org/0000-0001-6751-9091 


\author{
Young-Cheol Lee \\ https://orcid.org/0000-0001-6371-7445 \\ Hye-Jung Lee \\ https://orcid.org/0000-0001-5817-9566 \\ Gyeo-Woon Jung \\ https://orcid.org/0000-0002-1026-0290 \\ Na-Ra Yun \\ https://orcid.org/0000-0002-4219-0127 \\ Yo-Seob Seo \\ https://orcid.org/0000-0003-1804-5648 \\ $\mathrm{Ji}-\mathrm{Su} \mathrm{Oh}$ \\ https://orcid.org/0000-0002-8369-5025 \\ Jae-Seek You \\ https://orcid.org/0000-0001-7638-9583
}

\section{REFERENCES}

1. Carek PJ, Dickerson LM, Sack JL. Diagnosis and management of osteomyelitis. Am Fam Physician 2001;63:2413-2420.

2. Adekeye E0, Cornah J. Osteomyelitis of the jaws: a review of 141 cases. Br J Oral Maxillofac Surg 1985;23:24-35.

3. Koorbusch GF, Fotos P, Goll KT. Retrospective assessment of osteomyelitis. Etiology, demographics, risk factors, and management in 35 cases. Oral Surg Oral Med Oral Pathol 1992;74:149154.

4. Hudson JW. Osteomyelitis of the jaws: a 50-year perspective. J Oral Maxillofac Surg 1993;51:1294-1301.

5. Storoe W, Haug RH, Lillich TT. The changing face of odontogenic infections. J Oral Maxillofac Surg 2001;59:739-748; discussion 748-749.

6. Neville BW, Damm DD, Allen CM, Chi AC. Oral and maxillofacial pathology. 4th ed. St. Louis: Elsevier; 2016. pp. 128-135.

7. Renk H, Stoll L, Neunhoeffer F, et al. Suspicion of respiratory tract infection with multidrug-resistant Enterobacteriaceae: epidemiology and risk factors from a Paediatric Intensive Care Unit. BMC Infect Dis 2017;17:163.

8. Ersoz G, Uguz M, Aslan G, Horasan ES, Kaya A. Outbreak of meningitis due to Serratia marcescens after spinal anaesthesia. J Hosp Infect 2014;87:122-125.

9. Horcajada JP, Martínez JA, Alcón A, et al. Acquisition of multidrug-resistant Serratia marcescens by critically ill patients who consumed tap water during receipt of oral medication. Infect Control Hosp Epidemiol 2006;27:774-777.

10. Richards MJ, Edwards JR, Culver DH, Gaynes RP. Nosocomial in- fections in combined medical-surgical intensive care units in the United States. Infect Control Hosp Epidemiol 2000;21:510-515.

11. Burton DJ, Scheffer RB. Serratia infection in a patient with bilateral subcondylar impacted third molars and associated dentigerous cysts: report of case. J Oral Surg 1980;38:135-138.

12. Warren NP, Coombs RR. Delayed Serratia marcescens osteomyelitis following a gunshot injury. Injury 1991;22:493-494.

13. Lowe J, Kaplan L, Liebergall M, Floman Y. Serratia osteomyelitis causing neurological deterioration after spine fracture. A report of two cases. J Bone Joint Surg Br 1989;71:256-258.

14. Turgut N, Akgul T, Arzu U, et al. A rare pathogen for subacute osteomyelitis in adolescent: Serratia marcescens. Int J Surg Case Rep 2015;8C:171-174.

15. Lin TY, Chi HW, Wang NC. Pathological fracture of the right distal radius caused by Enterobacter aerogenes osteomyelitis in an adult. Am J Med Sci 2010;339:493-494.

16. Goaz PW, White SC. Infection and inflammation of the jaws and facial bones. In: Goaz PW, White SC, eds. Oral radiology: principles and interpretation. 3rd ed. St. Louis: Mosby; 1994. pp. 381397.

17. Bamberger DM. Osteomyelitis. A commonsense approach to antibiotic and surgical treatment. Postgrad Med 1993;94:177-182, 184.

18. Daramola JO, Ajagbe HA. Chronic osteomyelitis of the mandible in adults: a clinical study of 34 cases. Br J Oral Surg 1982;20:5862.

19. Ida M, Watanabe H, Tetsumura A, Kurabayashi T. CT findings as a significant predictive factor for the curability of mandibular osteomyelitis: multivariate analysis. Dentomaxillofac Radiol 2005;34:86-90

20. Schaberg DR, Culver DH, Gaynes RP. Major trends in the microbial etiology of nosocomial infection. Am J Med 1991;91:72S$75 \mathrm{~S}$.

21. Chevalier J, Mulfinger C, Garnotel E, Nicolas P, Davin-Régli A, Pagès JM. Identification and evolution of drug efflux pump in clinical Enterobacter aerogenes strains isolated in 1995 and 2003 PLoS One 2008;3:e3203.

22. Langley JM, Hanakowski M, Leblanc JC. Unique epidemiology of nosocomial urinary tract infection in children. Am J Infect Control 2001;29:94-98.

23. Mahlen SD. Serratia infections: from military experiments to current practice. Clin Microbiol Rev 2011;24:755-791.

24. Engel HJ, Collignon PJ, Whiting PT, Kennedy KJ. Serratia sp. bacteremia in Canberra, Australia: a population-based study over 10 years. Eur J Clin Microbiol Infect Dis 2009;28:821-824.

25. Acar JF. Serratia marcescens infections. Infect Control Hosp Epidemiol 1986;7:273-280.

26. Yu VL. Serratia marcescens: historical perspective and clinical review. N Engl J Med 1979;300:887-893. 